\title{
Rettung extrem: Einsatz in der Riesending-Höhle
}

\author{
Bettina Heberer
}

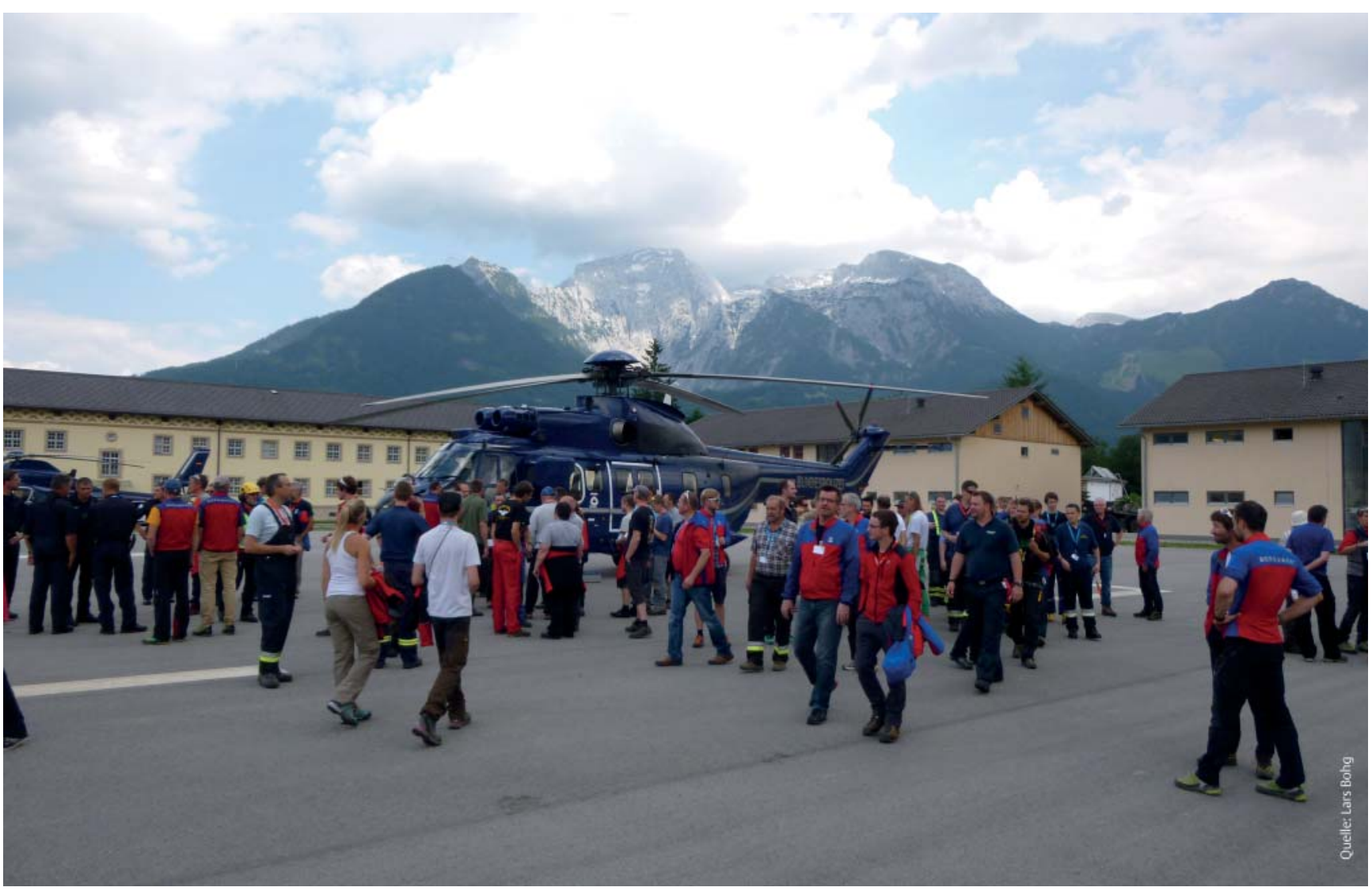

8. Juni 2014. In der Riesending-Höhle ereignet sich ein schwerer Unfall: Weit unter der Erde erleidet der Höhlenforscher Johann Westhauser bei einem Steinschlag schwere Kopfverletzungen. Ohne Arzt, ohne Funkkontakt, nicht ansprechbar liegt er in 1000 Metern Tiefe. Seine Begleiter entscheiden: Einer bleibt bei Westhauser. Die anderen machen sich an den Aufstieg durch das komplizierte Höhlensystem, um Hilfe zu holen.

\section{Der Einsatz}

\section{Notfallmeldung}

Noch am selben Tag um die Mittagszeit erreicht die Meldung über den Unfall Lars Bohg: Der 47-Jährige ist Geschäftsführer eines IT-Unternehmens in der Nähe von Stuttgart - und nebenberuflich ein erfahrener Höhlenforscher. Die Community ist klein, man kennt sich. Über eine eigene Rettungskette erfährt er bereits kurz nach dem Ausstieg von Westhausers Begleiter aus der Höhle von dem Unglück. „Ich war geschockt“, erzählt Bohg. „Ich bin mit Westhauser befreundet, wir haben die Höhle zusammen erforscht - und mir war klar, dass ein solcher Unfall lebensbedrohlich ist!“ Die Riesending-Höhle im
Untersberg bei Berchtesgaden ist die derzeit tiefste und längste bekannte Höhle Deutschlands. Es wäre schon schwierig, überhaupt medizinische Ausrüstung und genug Helfer zur Unfallstelle zu bringen.

Bohg telefoniert sofort mit einem Bekannten bei der Bergwacht und bietet seine Hilfe an. Ohne offizielle Beauftragung kann er jedoch zunächst nichts machen. Am Dienstagmorgen schickt die Bergwacht dann endlich eine SMS, ob er kommen kann. „Ich ging davon aus, dass ich in die Höhle soll, und habe meine gesamte Ausrüstung gepackt", so Bohg. In der Firma hinterlegt er die nötigen Passwörter, damit seine Mitarbeiter eine Weile ohne ihn zurechtkommen. Am Dienstagabend ist Bohg vor Ort. 


\section{Welche Fachärzte können zum \\ Verletzten - was können die \\ Höhlenforscher übernehmen?}

\section{Helfer und Material aus dem ganzen Alpengebiet}

Bohg geht davon aus, direkt in der Höhle den Einsatz zu unterstützen und bis zur Rettung unter der Erde zu bleiben. Es kommt aber anders: „Die Bergwachtleitstelle meinte, aus einsatztaktischen Gründen verzichte man vorerst auf meinen Einsatz in der Höhle“, erklärt er. Man bittet ihn, trotzdem vor Ort zu bleiben und die Einsatzkräfte mit seiner Ortskenntnis zu beraten. Es gibt jedoch Anlaufverzögerungen beim Rettungseinsatz. Viele Fragen sind zu klären: Wer kann in die Höhle, welche Fachärzte können zum Verletzten, und welche ärztlichen Aufgaben können erfahrene Höhlenforscher übernehmen? Wer kann ein Transportsystem aufbauen, um einen Schwerverletzten aus 1000 Metern Tiefe zu bergen? In Deutschland gibt es zwar eigene Rettungstrupps aus Höhlenforschern, die auch regelmäßige Rettungsworkshops durchführen, und jeder Höhlenforscher hat ein Erste-Hilfe-Set dabei. „Aber bei einer derartigen Verletzung sind die Möglichkeiten für einen medizinischen Laien sehr begrenzt“, erklärt Bohg.

Helfer aus dem ganzen Alpengebiet treffen ein. Einige Höhlenforscher aus Österreich, die sich in der Riesending-Höhle auskennen, zusammen mit einem Rettungssanitäter der deutschen Bergwacht bilden die Vorhut. Sie leisten die Erstversorgung des Verletzten mit Medikamenten und Kochsalzlösung, um eine völlige Dehydrierung zu verhindern. Nötiges Material in die enge, kalte und nasse Höhle zu bringen, dauert viele Stunden. „Es ist extrem anstrengend, sich in der Höhle zu bewegen“, erzählt Bohg. „Jeder Schritt muss überlegt sein und jeder Handgriff sitzen. Es ist oft eng und steil, man muss ständig hochkonzentriert sein."

\section{Die Bergung des Patienten ist für das Rettungsteam psychologisch sehr belastend.}

Bohg gibt Tipps, kann die verschiedenen Stellen in der Höhle einschätzen, kennt die Gefahren und weiß, wie viel Ausrüstung benötigt wird. Er unterstützt diejenigen, die in die Höhle gehen, auch mental. „Wenn Sie sich in einen $200 \mathrm{~m}$ tiefen Schacht abseilen und dabei in eine Tiefe wie vom Stuttgarter Fernsehturm runterschauen, ist das psychologisch sehr anspruchsvoll." Sehr positiv erlebt er die kurzen Entscheidungswege. Ein Beispiel: Die Einsatzkräfte sind zunächst auf dem Gelände der Feuerwehr Berchtesgaden untergebracht. „Dort hatte aber jeder Zugang, und es gab Probleme mit der Presse und nicht verifizierten Leuten." Nach wenigen Tagen wird das Lager daher in die Jägerkaserne der Bundeswehr in Bischofswiesen-Strub bei Berchtesgaden verlegt.

In der streng bewachten Kaserne ist das Rettungsteam unter sich und kann die Einrichtungen mit nutzen, von der Kantine bis zu Unterkünften. Die Anspannung bleibt trotzdem: „Ich habe am Anfang faktisch null geschlafen, weil ich immer mit meinem Einsatz gerechnet habe“, sagt Bohg. „Außerdem fühlte es sich an wie in einem Vietnamfilm: Tag und Nacht knatterten die Hubschrauber über meinem Bett. Das war sehr anstrengend.“

Nach ein paar Tagen ist für Bohg schließlich klar, dass er nicht in der Höhle zum Einsatz kommen wird. Er sieht, wie das Rettungsteam immer mehr Routine entwickelt das sorgt bei allen für eine gewisse Entspannung, und der Druck lässt nach. „Die große Hilfsbereitschaft unter den Rettern und Höhlenforschern war beeindruckend“, erinnert sich Bohg. „Man hat sich nie allein gefühlt, und es gab viele Gespräche. Die herzliche Aufnahme und die gute Integration haben allen geholfen, mit der Situation klarzukommen.“

\section{Transport aus dem Berg}

Nachdem Westhauser einigermaßen stabilisiert ist, muss geklärt werden, ob er noch in der Höhle operiert werden muss oder transportfähig ist. Neben einer eher harmlosen Platzwunde im Gesicht und einem Jochbeinbruch liegt höchstwahrscheinlich ein massives Schädel-HirnTrauma vor. Die Schwere des Schädel-Hirn-Traumas ordnen die Helfer zunächst mit einem standardisierten Testverfahren ein. Anhand dieser ersten zusammengestellten Daten über die Verletzungen wird das Fachärzteteam zusammengestellt. Da es unter den Höhlenforschern erstaunlich viele Ärzte gibt, greift die Bergwacht bevorzugt auf diese zurück, um eine Unfallgefahr so gering wie möglich zu halten.

Ein Schweizer Team aus Rettern, Höhlenforschern und Ärzten, das mittlerweile eingetroffen ist, testet die Transportfähigkeit des Verletzten. „Die Bergwacht hat wirklich alle mobilisiert, die erreichbar waren“, so Bohg. „Die Helfer mussten sich ja auch in der Höhle abwechseln und regelmäßige Arbeits- und Ruhezeiten einhalten, damit sich der Verletzte auf einen gleichmäßig wiederkehrenden Belastungs- und Ruherhythmus einstellen konnte.“

Die Ärzte schätzen Westhausers Zustand als stabil genug ein, um ihn aus der Höhle bringen zu können - aber sie dämpfen die Hoffnungen: „Die Ärzte meinten, wir könnten froh sein, wenn er überlebt“, sagt Bohg. „Viele gingen davon aus, dass er im Fall des Überlebens ein Schwerstpflegefall bleibt." Eine Ruhigstellung beispielsweise, wie sie normalerweise bei Patienten mit Schädel-Hirn-Trauma erfolgt, ist wegen des notwendigen Transports nicht möglich. 
Nachdem das Schweizer Rettungsteam die Transportfähigkeit Westhausers getestet und den Transport im ersten Schacht durchgeführt hat, kann fünf Tage nach dem Unfall der Abtransport zum Endschacht endlich beginnen. Dafür kommt ein italienischer Rettungstrupp zum Einsatz. „Die italienischen Kollegen haben deutlich mehr Erfahrung, und die italienische Bergwacht hat eigene Höhlenrettungstrupps“, erklärt Bohg. „In Italien ist Höhlenforschung eher ein Breitensport - entsprechend gibt es auch mehr Unfälle." Glücklicherweise gibt es keine Verständigungsprobleme: Einige Höhlenforscher sprechen Italienisch, Deutsch und Englisch, für die Einsatzbesprechungen stehen Übersetzer bereit. Unter Tage sprechen die Kollegen Italienisch, denn das italienische Team übernimmt einen Großteil der Rettungsaktion.

Täglich bekommt das Team in der Einsatzzentrale Informationen zum aktuellen Stand aus der Höhle und über die nächsten geplanten Schritte. Zwischen dem Basislager am Untersberg und der Kaserne kommunizieren die Teams über Funk. In der Höhle ist dies nicht möglich: Dort baut das Rettungsteam ein sogenanntes Cave-LinkSystem auf, über das eine SMS-ähnliche Kommunikation möglich ist.

Dem Verletzten geht es täglich etwas besser und die Chance steigt, ihn lebend aus der Höhle zu bekommen. Aber auch die Rettungsmannschaft ist nicht beliebig belastbar: 4 Stunden Arbeit wechseln mit 4 Stunden Ruhezeit, hinzu kommt die Nachtruhe. Ein Experte für Schädel-Hirn-Traumata, der auch Erfahrung mit Höhlenforschung hat, bespricht vor Ort, wie die Rettung theoretisch ablaufen sollte. Ein Vorteil bei der Rettungsaktion ist die verhältnismäßig leichte Zugänglichkeit der Stelle, an der sich der Verletzte befindet. „Es sind zwar weite Wege und große Schächte, aber es mussten keine Engstellen aufgesprengt werden“, erklärt Bohg. Trotzdem dauert der Transport durch die Höhle ganze 6 Tage. Währenddessen wird ein Hubschrauberlandeplatz geebnet und ein Untersuchungszelt aufgebaut.

\section{Zum Schluss droht noch ein Rettungs- kollaps.}

\section{Wie weit wird er sich erholen?}

Einen kritischen Moment gibt es noch kurz vor der finalen Rettung aus der Höhle: Es besteht die Gefahr eines Rettungskollapses, denn im 200 m langen Ein- und Ausstiegsschacht muss Westhauser senkrecht transportiert werden. Nach dem tagelangen Liegen auf der Trage riskiert man dabei Störungen der Herz-Kreislauf-Funktion und Hypothermie - erst recht bei der Umgebungstemperatur von $5{ }^{\circ} \mathrm{C}$ in der Höhle. „Für mich als medizinischen Laien war vorher nicht klar, dass es hier nochmal zu einer kritischen Situation kommen kann, in der ihnen der Verletzte quasi auf der Trage zum Hubschrauber wegstirbt“, sagt Bohg. Die Ärzte ziehen ihn noch einmal zu Rate, weil er Westhauser kennt und einschätzen kann, wie er sich verhalten wird.

Elf anstrengende und kritische Tage sind seit dem Unfall vergangen, als endlich die Bergung aus der Höhle gelingt. Die Ärzte versorgen Westhauser zunächst im Untersuchungszelt, bevor er mit einem bereitstehenden Hubschrauber in die Unfallklinik nach Murnau gebracht wird. Bereits nach einem Tag in der Klinik ist der Patient ansprechbar. An die Rettungsaktion selbst hat er nahezu keine Erinnerung, diese setzt erst im Hubschrauber bruchstückhaft ein.

\section{Rückblick}

Bohg erzählt: „Es gab nachher durchaus Kritik an dem Rettungseinsatz. Theoretisch wäre einiges sicher schneller gegangen - aber man unterschätzt leicht die praktischen Probleme vor Ort. Und im Nachhinein hat die Verzögerung wahrscheinlich sogar mehr genutzt als geschadet: Die lange Liegezeit bei kalten Temperaturen sowie die geringe Zufuhr von Nahrung und Flüssigkeit haben sich letztlich positiv ausgewirkt.“

Trotzdem leidet Westhauser noch längere Zeit unter den Nachwirkungen seiner Verletzungen, er ist oft schläfrig und hat Konzentrationsschwierigkeiten. „Bis er wieder zu $100 \%$ der Alte war, hat es sicher ein Jahr gedauert“, erinnert sich Bohg. „Aber dass er es überhaupt so weit geschafft hat, ist ein kleines medizinisches Wunder!“

\section{SPANNENDE EINSÄTZE}

Hatten auch Sie einen außergewöhnlichen Einsatz? Ob positiv oder negativ - retten! können Sie davon erzählen und Ihre Kollegen am konkreten Beispiel lernen lassen. Sie erreichen die Redaktion unter 07118931 -905 oder unter retten@thieme.de.

\section{KOMMENTAR}

von Dr. Sönke Müller, hausärztlicher Internist in Neckargemünd, Notarzt im Rettungsdienst und Mitherausgeber von retten!

Welchen Einsatz würden Sie verfilmen?

Der Rettungseinsatz in der Riesending-Höhle ist eine Steilvorlage für spektakuläre Drehbücher - und der Kontrast zum alltäglichen Rettungsdienst könnte kaum größer sein. Das gilt nicht nur für den Einsatzort, die Rettungsdauer oder die Zahl der beteiligten Retter. Da wird ein Höhlenforscher in 1000 m Tiefe von Steinen am Kopf getroffen, ist bewusstlos, nur noch ein Begleiter bleibt vor Ort. Der „normale Ret- 
tungsdienst“ kann nicht gerufen werden, weil der Weg zum Patienten unterirdisch verläuft und ca. $6 \mathrm{~km}$ lang ist. Alarmiert werden Rettungsdienste, Bergwachten, Höhlenforscher, zahlreiche Spezialisten - insgesamt 728 Retter aus 5 Nationen werden aktiv. Die Gesamtdauer der Rettung beträgt 11 Tage. In dieser Zeit entstehen u. a. ein neu gerodeter Hubschrauberlandeplatz und eine eigene Zeltstadt mit notfallmedizinischer Versorgungsstation; eine Bundeswehrkaserne wird zum Lager für die Einsatzkräfte, auch ein eigenes Pressezentrum entsteht - für die Live-Berichterstattung. Spektakulär sind auch die Gesamtkosten der Rettung: Rund 960000 Euro kostet die Aktion.

Wir alle kennen da eher den Stoff, aus dem die Drehbücher des Alltags sind. Meldungen auf unseren Piepsern, wie etwa: Radfahrer, männlich, Sturz auf der Straße, Ersthelfer vor Ort, Verdacht auf SHT. Die Leitstelle schickt einen RTW aus $5 \mathrm{~km}$ Entfernung sowie das nächste freie NEF vom Krankenhaus mit Notarztstandort (11 km Entfernung). Nach 7 min trifft der RTW ein, das NEF ist nach 12 min vor Ort. Und der Patient? Wurde von Ersthelfern in Seitenlage gebracht, liegt auf dem Grünstreifen neben der Landstraße. Somnolent, bewegt auf Aufforderung alles, Pupillenreaktion beidseitig normal, kreislauf- und atemstabil, ein paar Schürfungen am rechten Arm. Halsschiene, Vakuummatratze, Venenzugang, Monitoring als Basisversorgung. Schonende (aber zügige) Rettung von der Straße, Transport in die nächste chirurgische Klinik mit CT-Möglichkeit. Gesamte Einsatzdauer: ca. 50 min - routinemäßig erledigt von 3 Helden des Alltags. Medienecho? Evtl. am nächsten Tag in der Lokalpresse, als Fünf-Zeilen-Meldung in der Randspalte: „Radfahrer gestürzt“. Gesamtkosten des Einsatzes für die Krankenkasse: ca. $750-900 €$.

Lars Bohg war einer der Retter in der RiesendingHöhle und erlebte einen spektakulären Einsatz persönlich mit. Sein Bericht zeigt: Er war kein „Supermann“, kein „alleiniger Entscheider“ bei diesem Geschehen. Er war einer von sehr, sehr vielen Rettern einer, der dazu beitrug, dass die vielen kleinen Rädchen irgendwann ein großes und letztlich erfolgreiches (Rettungs-)Rad gedreht haben. So filmreif dieser Einsatz gewesen sein mag: Ein wenig Glanz davon strahlt vielleicht auch ab auf die vielen „No-Name-Helden“ des Alltags.
Bibliografie

doi:https://doi.org/10.1055/s-0043-105783 |

retten 2017; 6: 338-341

(c) Georg Thieme Verlag KG Stuttgart · New York ISSN 2193-2387

\section{Interessenkonflikt}

Die Autoren geben an, dass kein Interessenkonflikt besteht. 